Irish Math. Soc. Bulletin

Number 87, Summer 2021, 23-33

ISSN 0791-5578

\title{
On an inverse tangent problem
}

\author{
FINBARR HOLLAND AND ROGER SMYTH
}

\begin{abstract}
Even before the beginnings of Calculus a variety of methods for constructing tangents to plane curves were known. But what about the converse problemraised by Debaune in the seventeenth century: under what conditions will a given collection of straight lines be tangents to the same curve? Utilizing Hermite's interpolation theorem, we show in Section 2 that the members of any finite collection of lines are tangents to infinitely many differentiable plane curves. After first developing a prescient observation of Descartes in Section 3, we state and prove our main theorem in Section 4,. This describes sufficient conditions for a one-parameter collection of lines in complex form to be the family of tangents of a differentiable curve in the complex plane. As an application, we derive Jakob Steiner's nineteenth century result that, all save three members of the collection of Wallace-Simson lines of a triangle, are tangents to a deltoid whose incircle is the nine-point circle of the triangle.
\end{abstract}

\section{INTRODUCTION}

Since the time of Descartes (1596-1650) and Fermat (1601-1665) —and indeed long before [1] - a variety of methods have been developed for constructing tangents to plane curves whose equations were known in different coordinate systems, explicitly or implicitly. But what about the inverse problem? Knowing the tangents to a curve, is it possible to determine its equation? This problem appears to have been first raised by Florimond Debaune (1601-1652) ([2], p. 351), but mathematicians of the day were unable to solve it. While Descartes made a pertinent observation about the problem, which we develop in Section 3, it was left to Leibniz (1646-1716) to provide a satisfactory answer several decades later ([2], p. 426), one that ultimately led to the study of differential equations.

It is Debaune's inverse tangent problem that motivates the topic discussed here, but we treat a slightly different question. Precisely, we ask: under what conditions are members of a collection of straight lines in the complex plane $\mathbb{C}$ tangents to a differentiable curve? We begin by showing that a finite number of lines in $\mathbb{C}$ are tangents to infinitely many polynomials, and, guided by an observation made by Descartes in response to Debuane's question, proceed to present sufficient conditions under which members of a one-parameter collection of lines in $\mathbb{C}$ are tangents to a differentiable curve. We illustrate our methods by showing that all (save three) members of the collection of Wallace-Simson lines of a triangle are tangents to the deltoid that encloses the nine-point circle of the triangle, a result due to Jakob Steiner [8].

\section{A FINITE NUMBER OF LINES}

To keep the algebra to a minimum, we'll work throughout with the complex form of the equation of a straight line, rather than with its cartesian form. If $L$ is a straight

2020 Mathematics Subject Classification. 14H50, 51N25.

Key words and phrases. Tangent, curve, Hermite's interpolation formula, one-parameter family, Wallace-Simson lines, deltoid.

Received on 7-10-2020; revised 11-5-2021. 
line in the complex plane, its characteristic feature is that the unimodular expression

$$
t=\frac{a-b}{\bar{a}-\bar{b}}
$$

is the same for every pair of distinct points $a, b$ belonging to $L$. This invariant is called the clinant $^{1}$ of $L$-a useful term coined in 1890 by F. Franklin [3] and often cited in the work of Frank Morley ([5],[6]). Hence, if $z$ and $a$ belong to $L$, and are distinct, then $z-t \bar{z}=a-t \bar{a}$. Accordingly, the equation of a straight line in $\mathbb{C}$ can be described as the set of complex numbers $z$ that satisfy an equation of the form $z+\tau \bar{z}=c$, where $\tau$ and $c$ are constants, $\tau$ is a turn, i.e., a member of the unit circle $T$, and $\tau \bar{c}=c$; in which case $-\tau$ is the clinant of the line.

Given $n$ such lines in $\mathbb{C}$ with equations $z+\tau_{i} \bar{z}=c_{i}, i=1,2, \ldots, n$, where, for each subscript $i,\left|\tau_{i}\right|=1$, and $\tau_{i} \bar{c}_{i}=c_{i}$, we'll proceed to show that they are tangents to an analytic polynomial of degree $2 n-1$. Before doing so, however, it's convenient to recall Hermite's interpolation problem, which calls for a polynomial to have preassigned values and derivatives at specified places. To set the scene, select $n$ distinct (real or complex) numbers $x_{1}, x_{2}, \ldots, x_{n}$, and consider the problem of finding a polynomial $p$ such that $p\left(x_{i}\right)=a_{i}, p^{\prime}\left(x_{i}\right)=b_{i}, i=1,2, \ldots, n$, for preassigned real or complex numbers $a_{i}, b_{i}, i=1,2, \ldots, n$. Viewing this as a system of linear equations in the coefficients of $p$, and examining the matrix $M$ of coefficients, which is of Vandermonde's type, it's not too difficult to show that $|\operatorname{det} M|=\prod_{1 \leq i<j \leq n}\left|x_{i}-x_{j}\right|^{4}>0$. Hence, there is a unique polynomial $p$ of degree $2 n-1$ that interpolates the data. While this existence argument is sufficient for our purposes, it is useful to know Hermite's explicit formula for $p$. According to this, as can be readily verified,

$$
p(x)=\sum_{i=1}^{n}\left(a_{i}\left(\left(1-2 \pi_{i}^{\prime}\left(x_{i}\right)\left(x-x_{i}\right)\right)+b_{i}\left(x-x_{i}\right)\right) \pi_{i}(x)^{2},\right.
$$

where $\pi(x)=\prod_{i=1}^{n}\left(x-x_{i}\right)$, and

$$
\pi_{i}(x)=\frac{\prod_{j \neq i}\left(x-x_{j}\right)}{\pi^{\prime}\left(x_{i}\right)}, i=1,2, \ldots, n .
$$

In fact, this is a special case of a more general interpolation formula due to Spitzbart[7].

Returning to our tangent problem: for $i=1,2, \ldots, n$, denote by $u_{i}$ a square root of $-\tau_{i}$, take $a_{i}=c_{i} / 2, b_{i}=u_{i}$, and apply Hermite's result to obtain an analytic polynomial $p$ of degree $2 n-1$ such that

$$
p\left(x_{i}\right)=\frac{c_{i}}{2}, p^{\prime}\left(x_{i}\right)=u_{i}, i=1,2, \ldots, n
$$

Consider now the tangent to $p$ at the point $p\left(x_{i}\right)$; since $p^{\prime}\left(x_{i}\right) \neq 0$ it has equation $0=\Im\left\{\left(z-p\left(x_{i}\right)\right) \overline{p^{\prime}\left(x_{i}\right)}\right\}$. Inserting the values of $p$ and $p^{\prime}$ at $x_{i}$, this equation reduces to

$$
0=z-\frac{c_{i}}{2}-\left(\bar{z}-\frac{\bar{c}_{i}}{2}\right) \frac{u_{i}}{\bar{u}_{i}}
$$

But, by hypothesis, $\left|\tau_{i}\right|=1, \tau_{i} \bar{c}_{i}=c_{i}$, and $u_{i}^{2}=-\tau_{i}$, by choice. Hence, the latter form of the equation of the tangent becomes $z+\tau_{i} \bar{z}=c_{i}$, as required.

By way of illustration, we'll apply Hermite's formula to derive the equation of a quintic polynomial $p: \mathbb{R} \rightarrow \mathbb{C}$ that touches the three lines

$$
L_{1}: z+\bar{z}=-2 ; L_{2}: z+\bar{z}=0 ; L_{3}: z+\bar{z}=2 ;
$$

\footnotetext{
${ }^{1}$ Two lines are parallel iff they have the same clinant, and perpendicular, iff the sum of their clinants is zero,
} 
at the points $p(-1), p(0)$, and $p(1)$, respectively. These lines are parallel to the imaginary axis having the same clinant, viz., -1 , the square of $i$. Also, $c_{1}=-2, c_{2}=0$, and $c_{3}=2$. In addition, $x_{1}=-1, x_{2}=0$, and $x_{3}=1$, whence $\pi(x)=x\left(x^{2}-1\right)$ and

$$
\pi_{1}(x)=\frac{1}{2} x(x-1), \pi_{2}(x)=1-x^{2}, \pi_{3}(x)=\frac{1}{2} x(x+1) .
$$

Applying the formula, with $a_{1}=c_{1} / 2=-1, a_{2}=c_{2} / 2=0, a_{3}=c_{3} / 3=1$, and $b_{1}=b_{2}=b_{3}=i$, after some tedious calculation we get

$$
p(z)=\frac{1}{2}\left(3(i-1) z^{5}-5(i-1) z^{3}+2 i z\right), z \in \mathbb{R},
$$

as the desired polynomial, which can, of course, be verified directly.

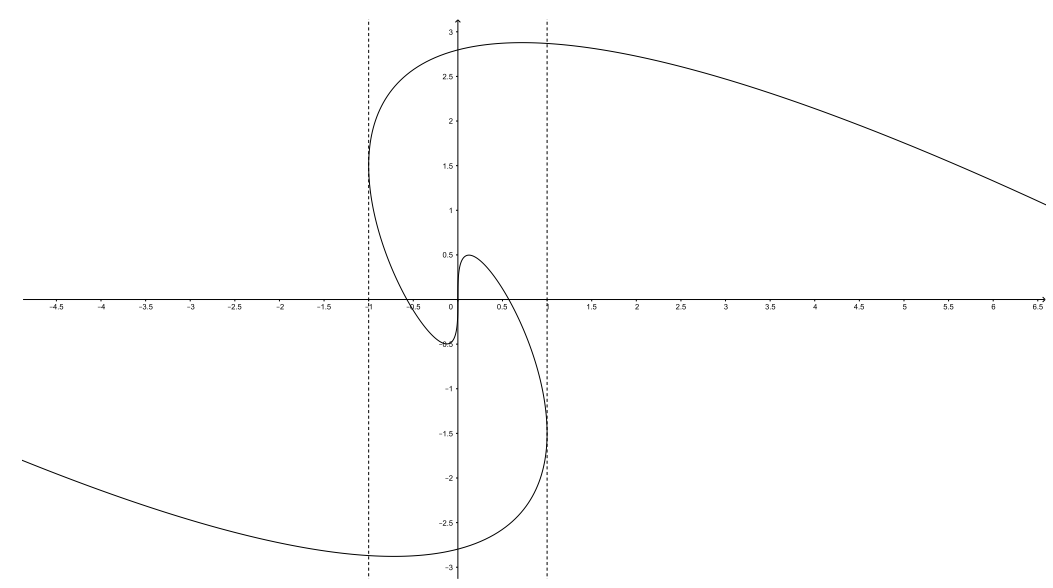

FIgURE 1. Quintic polynomial touching 3 parallel lines; $x=0, x= \pm 1$

It can also be easily verified that the concurrent lines

$$
M_{1}: z-i \bar{z}=0 ; M_{2}: z+\bar{z}=0 ; M_{3}: z+i \bar{z}=0 ;
$$

are tangents to the quintic

$$
\frac{1}{4} z\left(z^{2}-1\right)\left(u z(z-1)-4 i\left(1-z^{2}\right)+\bar{u} z(z+1)\right), z \in \mathbb{R},
$$

where $u:=(1+i) / \sqrt{2}$ is a square root of $i$, the clinant of $M_{1}$.

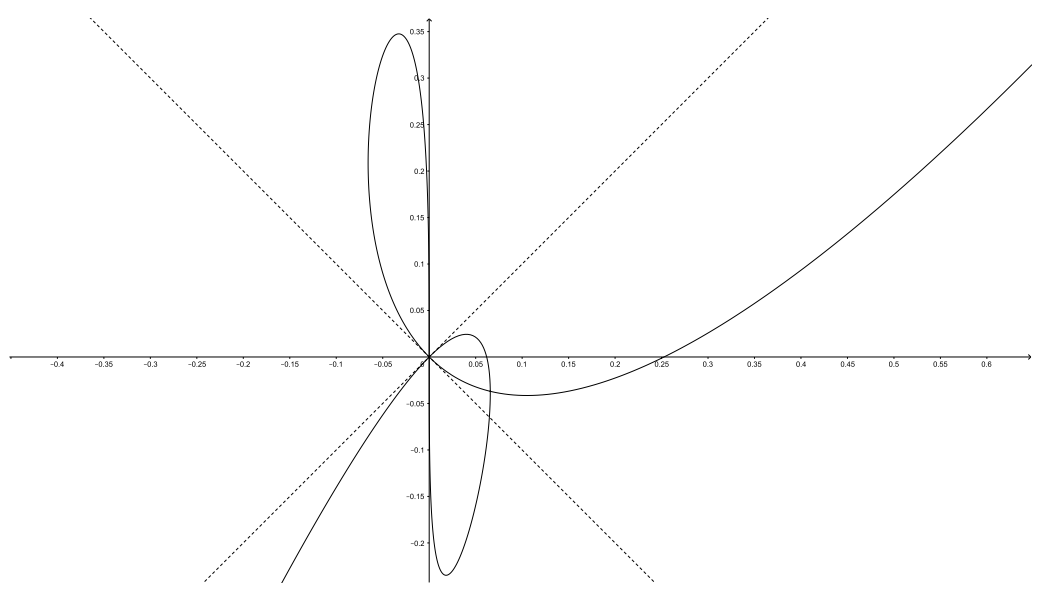

FIGURE 2. Quintic polynomial touching 3 concurrent lines; $x=0, y= \pm x$ 
A slight modification of the argument preceding these illustrative examples shows that the given lines $z+\tau_{i} \bar{z}=c_{i}, i=1,2, \ldots, n$, are also tangent to a trigonometric polynomial. To see this, select $n$ real numbers $\theta_{1}, \theta_{2}, \ldots, \theta_{n}$, so that the turns $x_{k}=$ $e^{i \theta_{k}}, k=1,2, \ldots, n$, are distinct. Keeping the same notation as before, determine the analytic polynomial $p$ of degree $2 n-1$ that satisfies the conditions

$$
p\left(x_{k}\right)=\frac{c_{k}}{2}, p^{\prime}\left(x_{k}\right)=i \bar{x}_{k} u_{k}, k=1,2, \ldots, n .
$$

Define the trigonometric polynomial $f$ on $(-\infty, \infty)$ by $f(x)=p\left(e^{i x}\right)$. If $f^{\prime}(x) \neq 0$, the equation of the tangent at $f(x)$ is given by

$$
z-\frac{f^{\prime}(x)}{\overline{f^{\prime}(x)}} \bar{z}=f(x)-\frac{f^{\prime}(x)}{\overline{f^{\prime}(x)}} \overline{f(x)} .
$$

In particular, since $f\left(\theta_{k}\right)=p\left(x_{k}\right)=\frac{c_{k}}{2}$, and $f^{\prime}\left(\theta_{k}\right)=i x_{k} p^{\prime}\left(x_{k}\right)=-u_{k} \neq 0$, the equation of the tangent at $f\left(\theta_{k}\right)$ is the set of $z$ such that

$$
\begin{aligned}
0 & =z-\frac{u_{k}}{\bar{u}_{k}} \bar{z}-\frac{c_{k}}{2}+\frac{u_{k}}{\bar{u}_{k}} \frac{\bar{c}_{k}}{2} \\
& =z-u_{k}^{2} \bar{z}-\frac{c_{k}}{2}+u_{k}^{2} \frac{\bar{c}_{k}}{2} \\
& =z+\tau_{k} \bar{z}-\frac{c_{k}+\tau_{k} \bar{c}_{k}}{2} \\
& =z+\tau_{k} \bar{z}-c_{k},
\end{aligned}
$$

since $\tau_{k} \bar{c}_{k}=c_{k}$, by hypothesis. Thus the family of lines $z+\tau_{i} \bar{z}=c_{i}, i=1,2, \ldots, n$, are tangents to $f$, a $2 \pi$-periodic function.

It follows from this that any $n$ straight lines are tangents to infinitely many analytic polynomials of degree $2 n-1$, and also to infinitely many trigonometric polynomials of degree $2 n-1$. The latter means, in particular, that the lines are tangents to many closed curves in $\mathbb{C}$.

Contrast this statement with the fact that three non-concurrent lines, no two of which are parallel, are tangents to precisely four circles, namely, the incircle and the three excircles of the triangle determined by the lines, something we learn in secondary school. For instance, the lines

$$
z-\bar{z}=0 ; z+i \bar{z}=1+i ; z-i \bar{z}=-1+i
$$

are tangents to the four circles

$$
\left.C_{1}:|z-(\sqrt{2}-1) i|=\sqrt{2}-1 ; C_{2}: \mid z-\sqrt{2}-i\right)\left|=1 ; C_{3}:\right| z+\sqrt{2}-i \mid=1 ;
$$

and $C_{4}:|z+(\sqrt{2}+1) i|=\sqrt{2}+1$. Of these, $C_{1}$ is the incircle of the triangle with vertices $-1,1$, and $i$, and $C_{2}, C_{3}$ and $C_{4}$ are its excircles.

This raises the possibility, that, by imposing suitable incidence relations on a set of $n$ lines, it may be possible to produce a finite number of closed curves to which some or all of the lines are tangents. Our intention is to explore this possibility in a future publication, whose purpose is to complement the approach taken in [5], where it is shown that finitely many curves of a certain kind touch $n$ lines.

\section{Descartes' insight}

We learn from ([2], p. 426), that Descartes gave the following response to Debeaune about the latter's inverse tangent problem mentioned in Section 1: "I do not believe that it is in general possible to find the converse to my rule of tangents, nor of that which M. Fermat uses, ...". But, on the same page, he leaves the following insightful remark to posterity: "There is indeed another method that is more general and a priori, namely, by the intersection of two tangents, which should always intersect between the 
points at which they touch the curve, as near one another as you can imagine; for in considering what the curve ought to be, in order that this intersection may occur between the two points, and not on that side or the other, the construction for it may be found."

This statement appears to apply in particular to the graphs of real convex (or concave) functions defined on subintervals of the real axis, and one can present sufficient conditions for it to hold for parametrically defined functions. What follows is our interpretation of what we believe Descartes may have had in mind.

Theorem 3.1. Suppose $I$ is a subinterval of $(-\infty, \infty)$ and $\gamma: I \rightarrow \mathbb{C}$ is twice continuously differentiable on $I$, and determines a curve $\Gamma$ with non-zero curvature at a point $u \in I$. Then there exists a neighbourhood $N$ of $u$ such that if $s, t \in N$ and $s \neq t$, the tangents $L_{t}$ and $L_{s}$ to $\Gamma$ at $\gamma(t)$ and $\gamma(s)$, respectively, intersect at a unique point $z(t, s)$, say, and

$$
\lim _{t \rightarrow u} z(t, u)=\gamma(u)
$$

Proof. By hypothesis, $\gamma$ is differentiable on $I$ and its derivative doesn't vanish there. Therefore the equation of the tangent to $\Gamma$ at any point $\gamma(t)$ is given by the set of $z$ such that $\Im\left\{(z-\gamma(t)) \overline{\gamma^{\prime}(t)}\right\}=0$, equivalently, $z+\tau(t) \bar{z}=c(t)$, where

$$
\tau(t)=-\frac{\gamma^{\prime}(t)}{\overline{\gamma^{\prime}(t)}}, \text { and } c(t)=\gamma(t)+\tau(t) \bar{\gamma}(t)
$$

Hence

$$
-\tau^{\prime}(t)=\frac{\gamma^{\prime \prime}(t) \overline{\gamma^{\prime}(t)}-\gamma^{\prime}(t) \overline{\gamma^{\prime \prime}(t)}}{\overline{\gamma^{\prime}(t)^{2}}}=\frac{2 i \Im\left(\gamma^{\prime \prime}(t) \overline{\gamma^{\prime}(t)}\right)}{\overline{\gamma^{\prime}(t)^{2}}} .
$$

This expression is continuous and non-zero at $u$, by assumption. Hence, by continuity, at least one of $\Re \tau^{\prime}, \Im \tau^{\prime}$ is non-zero on some neighbourhood $N$ of $u$. Hence, by the Mean Value Theorem, at least one of $\Re \tau, \Im \tau$ is one-one on $N$, whence $\tau$ is one-one on $N$. Consequently, if $t, s \in N$, and $t \neq s$, the corresponding tangents $L_{t}, L_{s}$ intersect and their point of intersection $z(t, s)$ is given by

$$
\bar{z}(t, s)=\frac{c(t)-c(s)}{\tau(t)-\tau(s)} .
$$

Clearly,

$$
\begin{aligned}
\lim _{t \rightarrow u} \bar{z}(t, u) & =\frac{c^{\prime}(u)}{\tau^{\prime}(u)} \\
& =\frac{\gamma^{\prime}(u)+\tau(u) \overline{\gamma^{\prime}(u)}+\tau^{\prime}(u) \bar{\gamma}(u)}{\tau^{\prime}(u)} \\
& =\frac{\tau^{\prime}(u) \bar{\gamma}(u)}{\tau^{\prime}(u)} \\
& =\bar{\gamma}(u) .
\end{aligned}
$$

In other words, the claim is true.

This result seems to be the basis for the recipe utilized by various authors who seek to determine the equation of a curve from a one-parameter set of lines they assume are its tangents. 


\section{ONE-PARAMETER FAMILIES OF LINES}

As we've seen, the members of any finite collection of straight lines are tangents to infinitely many curves. However, this no longer holds if the collection is infinite. For instance, it's easy to see that the members of the one-parameter family of lines indexed by $t$ on $[0, \infty)$, with cartesian equations $y+t x=1$, are not all tangents to the same planar curve defined on $[0, \infty)$. In this section, we prescribe sufficient conditions for all or some members of a one-parameter family of lines in $\mathbb{C}$ to be tangents to the same curve.

Definition 4.1. We call a pair of functions $\phi, \psi$ defined on an interval $I$ of the real line compatible on $I$, if, for all $t \in I,|\phi(t)|=1$ and $\phi(t) \overline{\psi(t)}=\psi(t)$.

For instance, the members of each of the ordered pairs $(1, \cos x),((i x-1) /(i x+$ $1), i /(i x+1))$, and $\left(e^{i n x},\left(1+e^{i x}\right)^{n}\right)$, where $n$ is a nonnegative integer, are compatible on any subinterval of $(-\infty, \infty)$.

Such a pair of compatible functions defines a one-parameter family of lines indexed on $I$, with equations $z+\phi(t) \bar{z}=\psi(t), t \in I$. Under what circumstances will such a pair generate lines some or all of which are tangents to a differentiable curve parameterised on $I$ ?

To get a handle on this problem, notice as before that if $f: I \rightarrow \mathbb{C}$ is differentiable and $t \in I$, then the curve $\Gamma_{f}=f(I)$, has a tangent $T_{t}$ at $f(t)$ as long as $f^{\prime}(t) \neq 0$, in which case its equation is the set of $z$ such that $\Im\left\{(z-f(t)) \overline{f^{\prime}(t)}\right\}=0$, equivalently, $z+\tau_{f}(t) \bar{z}=c_{f}(t)$, where

$$
\tau_{f}(t)=-\frac{f^{\prime}(t)}{\overline{f^{\prime}(t)}} \text { and } c_{f}(t)=f(t)+\tau_{f}(t) \overline{f(t)} .
$$

Clearly, $\tau_{f}$ and $c_{f}$ are compatible on $I$. Consequently, if, for some compatible pair $\phi, \psi$ on $I$, and some $t \in I$, the equation $z+\phi(t) \bar{z}=\psi(t)$ coincides with that for $T_{t}$, then $\phi(t)=\tau_{f}(t)$ and $\psi(t)=c_{f}(t)$, so that

$$
\overline{f^{\prime}(t)} \phi(t)+f^{\prime}(t)=0 \text {, and } f(t)+\overline{f(t)} \phi(t)=\psi(t) .
$$

Conversely, if for a given pair of compatible functions these functional equations are satisfied by an appropriate function $f$ at some point $t \in I$, the set of $z$ such that $z+\phi(t) \bar{z}=\psi(t)$ is the tangent to $\Gamma_{f}$ at $f(t)$.

To consider further the solution $f$ of these last displayed equations, assume $\phi, \psi$ are differentiable on $I$. Then, by differentiation of the second equation, and using the first, we see that

$$
\begin{aligned}
\psi^{\prime}(t) & =\frac{d}{d t}(f(t)+\overline{f(t)} \phi(t)) \\
& =f^{\prime}(t)+\overline{f^{\prime}(t)} \phi(t)+\overline{f(t)} \phi^{\prime}(t) \\
& =\overline{f(t)} \phi^{\prime}(t) .
\end{aligned}
$$

In other words, at least formally,

$$
f(t)=\frac{\overline{\psi^{\prime}(t)}}{\overline{\phi^{\prime}(t)}}
$$

This formula, backed up by Theorem 3.1, suggests a means of recovering the equation of a curve some or all of whose tangents are assumed to be of the form $z+\bar{z} \phi(t)=\psi(t)$, for some $t \in I$, where $\phi, \psi$ are at least compatible and possess certain differentiability properties, as yet unstated. The next theorem supports this statement. 
Theorem 4.2. Suppose $\phi, \psi$ are compatible on I, twice differentiable there and such that $\phi^{\prime}$ and $\psi^{\prime \prime} \phi^{\prime}-\psi^{\prime} \phi^{\prime \prime}$ are both non-zero on $I$. Then the collection of lines $L_{t}$ : $z+\phi(t) \bar{z}=\psi(t), t \in I$, coincides with the family of tangents to a differentiable curve $C$ parameterised on I by the complex conjugate of $\psi^{\prime} / \phi^{\prime}$.

Proof. Define $f$ to be the complex conjugate of $\psi^{\prime} / \phi^{\prime}$. Let $C=f(I)$. Since by hypothesis, $\phi^{\prime} \neq 0, f$ is well-defined on I and differentiable there with derivative given by

$$
\bar{f}^{\prime}=\frac{\psi^{\prime \prime} \phi^{\prime}-\psi^{\prime} \phi^{\prime \prime}}{\left(\phi^{\prime}\right)^{2}}
$$

which is non-zero on $I$ by assumption. Hence, the tangent $T$ to $C$ at $f(t)$ has equation $z+\tau(t) \bar{z}=c(t)$, where $\tau(t)=-\frac{f^{\prime}(t)}{f^{\prime}(t)}$ and $c(t)=f(t)+\tau(t) \overline{f(t)}$.

We claim that $T$ coincides with $L_{t}$. Since $\phi \bar{\psi}=\psi$, and $\frac{d}{d x} \bar{g}=\overline{g^{\prime}}$ for any differentiable function $g$ on $(-\infty, \infty)$, we have that $\phi^{\prime} \bar{\psi}+\phi \bar{\psi}^{\prime}=\psi^{\prime}$, whence

$$
\bar{\psi}+\frac{\phi \bar{\psi}^{\prime}}{\phi^{\prime}}=\bar{f}, \text { and so } \phi \bar{f}=\phi \bar{\psi}+\frac{\phi^{2} \bar{\psi}^{\prime}}{\phi^{\prime}}=\psi+\frac{\phi^{2} \bar{\phi}^{\prime} f}{\phi^{\prime}} .
$$

Hence

$$
f+\phi \bar{f}=\psi+\left(1+\frac{\phi^{2} \bar{\phi}^{\prime}}{\phi^{\prime}}\right) f=\psi
$$

because $1=\phi \bar{\phi}$ and so $0=\phi^{\prime} \bar{\phi}+\phi \overline{\phi^{\prime}}$. Hence, in particular, $f(t)+\phi(t) \overline{f(t)}=\psi(t)$, which means that $f(t) \in L_{t}$. Next, we prove that $T$ and $L_{t}$ have the same clinants. The claim is that $f^{\prime}(t)+\phi(t) \overline{f^{\prime}(t)}=0$. But, as we've just seen, $f+\phi \bar{f}=\psi$, hence $f^{\prime}+\phi \overline{f^{\prime}}+\phi^{\prime} \bar{f}=\psi^{\prime}=\phi^{\prime} \bar{f}$, which means that $f^{\prime}+\phi \overline{f^{\prime}}=0$, and so, in particular, the claim is true. Thus, $L_{t}$ and $T$ are parallel, and so coincident, since they share the point $f(t)$.

Example 4.3. All but one of the lines

$$
z+e^{3 i x} \bar{z}=\left(1+e^{i x}\right)^{3},
$$

parameterised on $[0,2 \pi]$, is a tangent to the cardioid curve $z(x)=\left(1+e^{i x}\right)^{2}$.

Proof. The family of given lines is generated by the compatible functions $\phi(x)=e^{3 i x}$, and $\psi(x)=\left(1+e^{i x}\right)^{3}$. Also, $\phi^{\prime} \neq 0$ and

$$
\frac{\psi^{\prime}(x)}{\phi^{\prime}(x)}=\frac{3 i e^{i x}\left(1+e^{i x}\right)^{2}}{3 i e^{3 i x}}=\left(1+e^{-i x}\right)^{2},
$$

so that

$$
\psi^{\prime \prime}(x) \phi^{\prime}(x)-\psi^{\prime}(x) \phi^{\prime \prime}(x)=\phi^{\prime}(x)^{2}\left(\frac{\psi^{\prime}(x)}{\phi^{\prime}(x)}\right)^{\prime}=-2 i \phi^{\prime}(x)^{2} e^{-i x}\left(1+e^{-i x}\right) .
$$

Hence $\psi^{\prime \prime}(x) \phi^{\prime}(x)-\psi^{\prime}(x) \phi^{\prime \prime}(x)$ is non-zero save at $x=\pi$. Hence Theorem 4.2 applies on each of the intervals $[0, \pi),(\pi, 2 \pi]$, and the stated result follows.

Example 4.4. Suppose $|\alpha| \neq 1$. All the lines

$$
z+\frac{e^{2 i x}-\alpha}{1-\bar{\alpha} e^{2 i x}} \bar{z}=\frac{e^{i x}}{1-\bar{\alpha} e^{2 i x}},
$$

parameterised on $[0,2 \pi]$, are tangents to an ellipse. 
Proof. To begin with, it's not too difficult to see that the differentiable functions $\phi, \psi$ defined on $[0,2 \pi]$ by

$$
\phi(x)=\frac{e^{2 i x}-\alpha}{1-\bar{\alpha} e^{2 i x}}, \psi(x)=\frac{e^{i x}}{1-\bar{\alpha} e^{2 i x}},
$$

are compatible, satisfy the conditions of Theorem 4.2, and that

$$
\phi^{\prime}(x)=\frac{2 e^{i x}\left(1-|\alpha|^{2}\right)}{\left(1-\bar{\alpha} e^{2 i x}\right)^{2}}, \psi^{\prime}(x)=\frac{1+\bar{\alpha} e^{2 i x}}{\left(1-\bar{\alpha} e^{2 i x}\right)^{2}},
$$

Hence the given lines are tangents to the curve defined by

$$
f(x)=\frac{e^{i x}+\alpha e^{-i x}}{2\left(1-|\alpha|^{2}\right)} .
$$

which describes an ellipse.

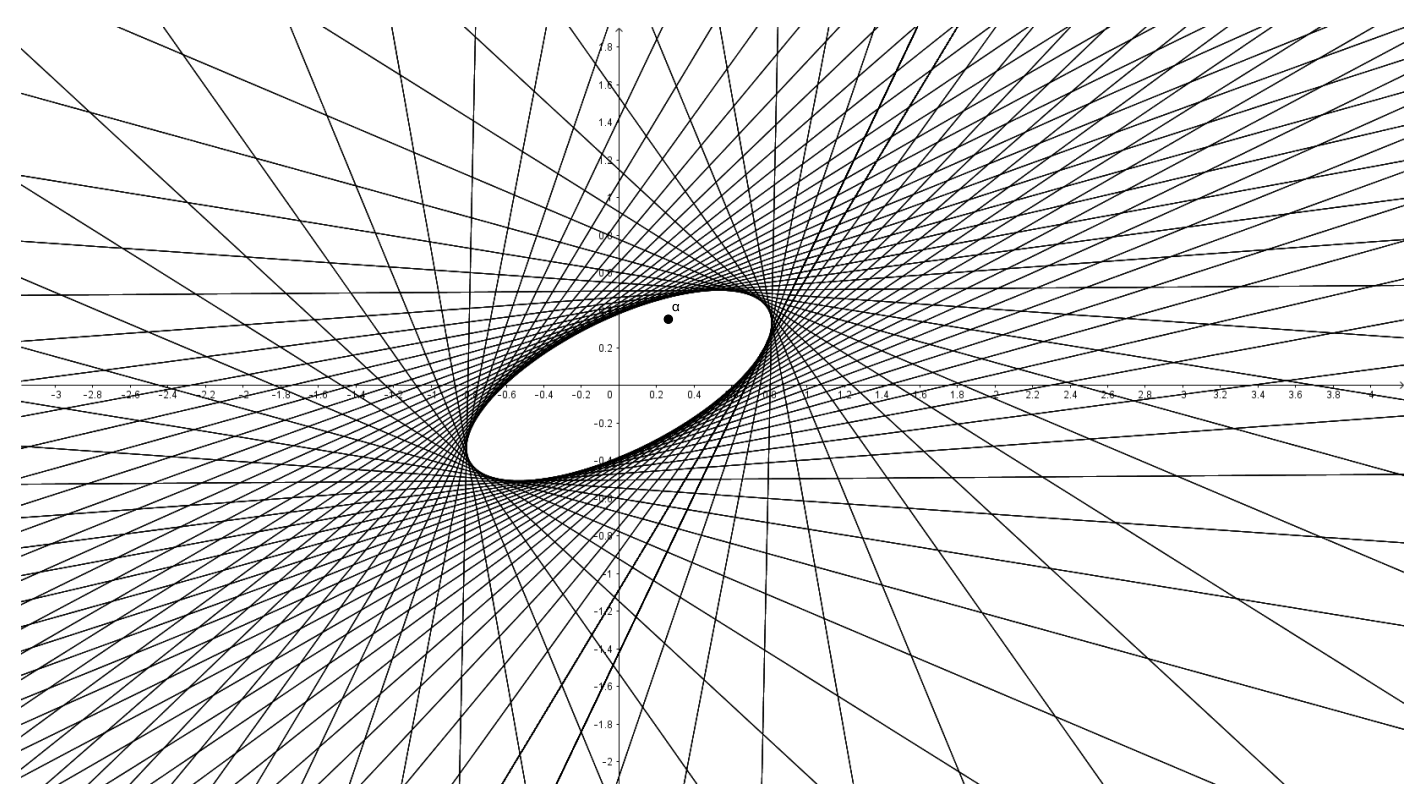

Figure 3. A family of lines that touch an ellipse

\section{WALlace-Simson lines of a triangle}

Suppose the numbers $t_{1}, t_{2}$, and $t_{3}$ are distinct turns. Consider them to be the vertices of a triangle $A B C$ inscribed in the unit circle $T$, and denote by $s_{1}, s_{2}$, and $s_{3}$ their corresponding symmetric polynomials. The three equations

$$
z+t_{1} t_{2} \bar{z}=t_{1}+t_{2}, z+t_{2} t_{3} \bar{z}=t_{2}+t_{3}, z+t_{3} t_{1} \bar{z}=t_{3}+t_{1}
$$

are those of the sides of $A B C$. If $|t|=1$, it's easy to verify that the numbers $p, q, r$, defined by

$$
2 p t=t^{2}+\left(t_{1}+t_{2}\right) t-t_{1} t_{2}, 2 q t=t^{2}+\left(t_{2}+t_{3}\right) t-t_{2} t_{3}, 2 r t=t^{2}+\left(t_{3}+t_{1}\right) t-t_{3} t_{1},
$$

are the projections from $t$ onto these lines. (For instance, $p$ is on the line $z+t_{1} t_{2} \bar{z}=$ $t_{1}+t_{2}$, and $t_{1} t_{2}$ is the clinant of the line joining $t$ and $p$.) Also,

$$
2 t(p-q)=\left(t_{1}-t_{3}\right) t-t_{1} t_{2}+t_{2} t_{3}=\left(t_{1}-t_{3}\right)\left(t-t_{2}\right),
$$

so that

$$
\frac{p-q}{\bar{p}-\bar{q}}=\bar{t} t_{1} t_{2} t_{3}=\bar{t} s_{3} .
$$


Hence, $\bar{t} s_{3}$ is the clinant of the line through $p$ and $q$. By symmetry, this is also the clinant of the line through $q$ and $r$. Hence, the points $p, q$, and $r$ are collinear, and lie on the line $W S(t)$, one form of whose equation is

$$
z-\bar{t} s_{3} \bar{z}=p-\bar{t} s_{3} \bar{p}
$$

This line is called the Wallace-Simson line of $A B C$ associated with $t$.

5.1. The Steiner deltoid of $A B C$. Inserting the value of $p$ given above, an alternative form of the equation for $W S(t)$ follows, namely,

$$
z-\bar{t} s_{3} \bar{z}=\frac{1}{2}\left(t+s_{1}-s_{2} \bar{t}-s_{3} \bar{t}^{2}\right) .
$$

Writing $\phi(t)=-\bar{t} s_{3}$ and $\psi(t)=p-\bar{t} s_{3} \bar{p}=p+\phi(t) \bar{p}$, observe that $|\phi(t)|=1$, and so

$$
\phi(t) \overline{\psi(t)}=\phi(t) \bar{p}+\phi(t) \overline{\phi(t)} p=|\phi(t)|^{2} p+\phi(t) \bar{p}=p+\phi(t) \bar{p}=\psi(t) .
$$

Hence, the infinitely differentiable functions $\phi\left(e^{i x}\right), \psi\left(e^{i x}\right)$ are compatible on $(-\infty, \infty)$. The equations

$$
z+\phi(x) \bar{z}=\psi(x), 0 \leq x \leq 2 \pi,
$$

therefore determine the one-parameter family of Wallace-Simson lines of $A B C$. Utilizing Theorem 4.2 we'll show that all but three members of this family are tangents to a three-cusped hypocycloid. To this end, note that

$$
\phi(x)=-e^{-i x} s_{3}, \psi(x)=\frac{1}{2}\left(e^{i x}+s_{1}-s_{2} e^{-i x}-s_{3} e^{-2 i x}\right),
$$

and so

$$
\phi^{\prime}(x)=i e^{-i x} s_{3}, \psi^{\prime}(x)=\frac{1}{2}\left(i e^{i x}+i s_{2} e^{-i x}+2 s_{3} i e^{-2 i x}\right) .
$$

Hence

$$
\frac{\psi^{\prime}(x)}{\phi^{\prime}(x)}=\frac{e^{2 i x}+s_{2}+2 s_{3} e^{-i x}}{2 s_{3}}=\frac{1}{2}\left(\bar{s}_{3} e^{2 i x}+\bar{s}_{1}+2 e^{-i x}\right),
$$

since $s_{2}=\bar{s}_{1} s_{3}$. According to Theorem 4.2, the function $f$ whose tangents are among those of the given family of Wallace-Simson lines is given by

$$
f(x)=\frac{1}{2}\left(s_{3} e^{-2 i x}+s_{1}+2 e^{i x}\right), 0 \leq x \leq 2 \pi,
$$

which is the equation of a deltoid, a closed curve with three cusps, resembling a curvilinear equilateral triangle. Alternatively, it can be viewed as the image of the unit circle under the map

Since

$$
z_{S}(t)=\frac{1}{2}\left(s_{1}+2 t+s_{3} \vec{t}^{2}\right)=\frac{t_{1}+t_{2}+t_{3}}{2}+t+\frac{t_{1} t_{2} t_{3}}{2} \vec{t}^{2},|t|=1 .
$$

$$
f^{\prime}(x)=-i e^{-2 i x}\left(e^{3 i x}-s_{3}\right)=-i e^{-2 i x}\left(e^{3 i x}-t_{1} t_{2} t_{3}\right),
$$

$f$ fails to have tangents at only its three cusp points, namely the points $z_{S}(\alpha), z_{S}(\beta), z_{S}(\gamma)$, where the turns $\alpha, \beta, \gamma$ are the distinct cube roots of $t^{3}-s_{3}$.

Notice that the constant term $\frac{1}{2} s_{1}=\frac{1}{2}\left(t_{1}+t_{2}+t_{3}\right)$ in the equation of $f$ is the centre of the nine-point circle associated with $A B C$, whose equation is $\left|z-\frac{s_{1}}{2}\right|=\frac{1}{2}$. Hence, if $|t|=1$, the point $\left(s_{1}+2 t+s_{3} \bar{t}^{2}\right) / 2$ on the deltoid lies on the nine-point circle iff

$$
\left|2 t+s_{3} \vec{t}^{2}\right|=1 \text {, i.e., }\left|2 t^{3}+s_{3}\right|=1,
$$

the solutions of which satisfy $t^{3}=-s_{3}$. This is so because if $|u|=1$, then $|2 u+1|=1$ iff $u=-1$. Thus, the nine-point circle of $A B C$ touches the deltoid at three points. Moreover, for all $t \in T$,

$$
\left|z_{S}(t)-\frac{s_{1}}{2}\right|=\frac{1}{2}\left|2 t+s_{3} \vec{t}^{2}\right| \geq \frac{1}{2}|2| t|-| s_{3} \vec{t}^{2}||=\frac{1}{2} .
$$


Therefore the nine-point circle of a triangle is inscribed in the deltoid generated by its family of Wallace-Simson lines. We refer to this deltoid as the Steiner deltoid of $A B C$ in honour of Jakob Steiner who discovered these results in the nineteenth century [8]; and denote it by $\delta_{S}$.

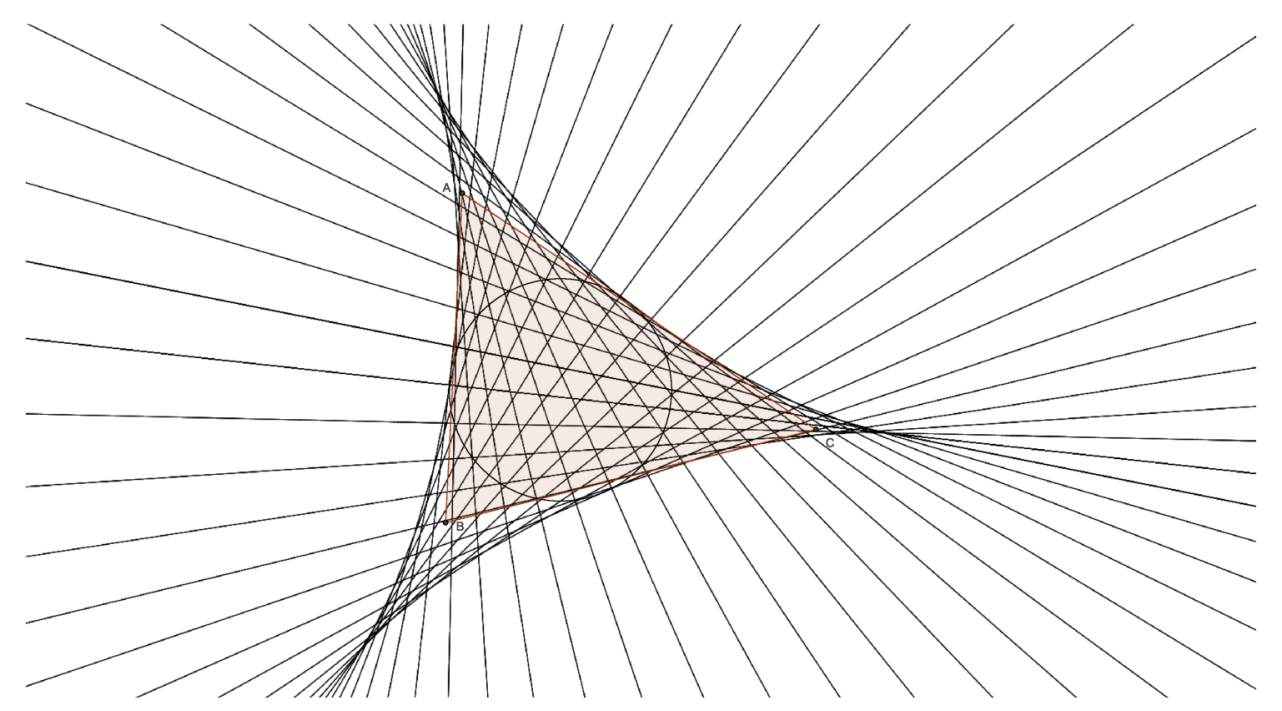

Figure 4. Triangle, 9 point circle, and Steiner deltoid

5.2. An outer deltoid of $A B C$. This section was motivated by the article [4].

Consider the line through a turn $t$ that is parallel to $W S(t)$, its Wallace-Simson line with respect to the same triangle $A B C$. Since the clinant of $W S(t)$ is $\bar{t} s_{3}$, the equation of the line under discussion has equation $z-\bar{t} s_{3} \bar{z}=t-s_{3} \bar{t}^{2}$. Therefore, as $t$ varies in $T$, another one-parameter family of such lines is generated, some or all of which are tangent lines to a curve whose equation can be found by appealing to Theorem 4.2 once more. Indeed, setting $\phi(x)=-e^{-i x} s_{3}, \psi(x)=e^{i x}-s_{3} e^{-2 i x}$, the curve in question can be seen to have equation $f(x)=2 e^{i x}+s_{3} e^{-2 i x} \equiv z_{o}\left(e^{i x}\right)$, where $z_{o}$ is defined on $T$ by $z_{o}(t)=2 t+s_{3} t^{2},|t|=1$. This curve is another deltoid, $\delta_{o}$, say, whose cusps occur at the roots on $T$ of the cubic $t^{3}-s_{3}$. Observe also that

$$
\left|z_{o}(t)\right| \geq|| 2 t|-| s_{3}|| t^{2}||=|2-1|=1,
$$

for all $t \in T$. Hence, $\delta_{o}$ encloses $T$ and touches it at some point $t \in T$, iff $\left|2 t^{3}+s_{3}\right|=1$, i.e., iff $t^{3}=-s_{3}$. In other words, $T$ is the inscribed circle of $\delta_{o}$, which touches it at precisely three points. Thus, $\delta_{o}$ encloses $A B C$, and touches its circumscribing circle $T$ at three points. We refer to $\delta_{o}$ as an outer deltoid associated with $A B C$. Since $2 z_{S}=s_{1}+z_{0}$, it's evident that $\delta_{S}$ is a translate of a scaled version of $\delta_{o}$. Clearly, tangents to $\delta_{S}$ are parellel to those of $\delta_{o}$.

For example, if $t_{1}=-1, t_{2}=1$, and $t_{3}=i$, so that $s_{1}=i, s_{2}=-1$, and $s_{3}=-i$, $A B C$ is a right-angled triangle, whose nine-point circle is $\left|z-\frac{i}{2}\right|=\frac{1}{2}$, whose WallaceSimson lines have equations $z+i \bar{t} \bar{z}=\frac{1}{2}\left(i+t+\bar{t}+i \bar{t}^{2}\right), t \in T$, and whose Steiner deltoid and outer deltoid, respectively, shown in the accompanying diagram, have equations $z_{S}(t)=\frac{1}{2}\left(i+2 t-i \vec{t}^{2}\right)$ and $z_{o}(t)=2 t-i \vec{t}^{2}$, respectively. 


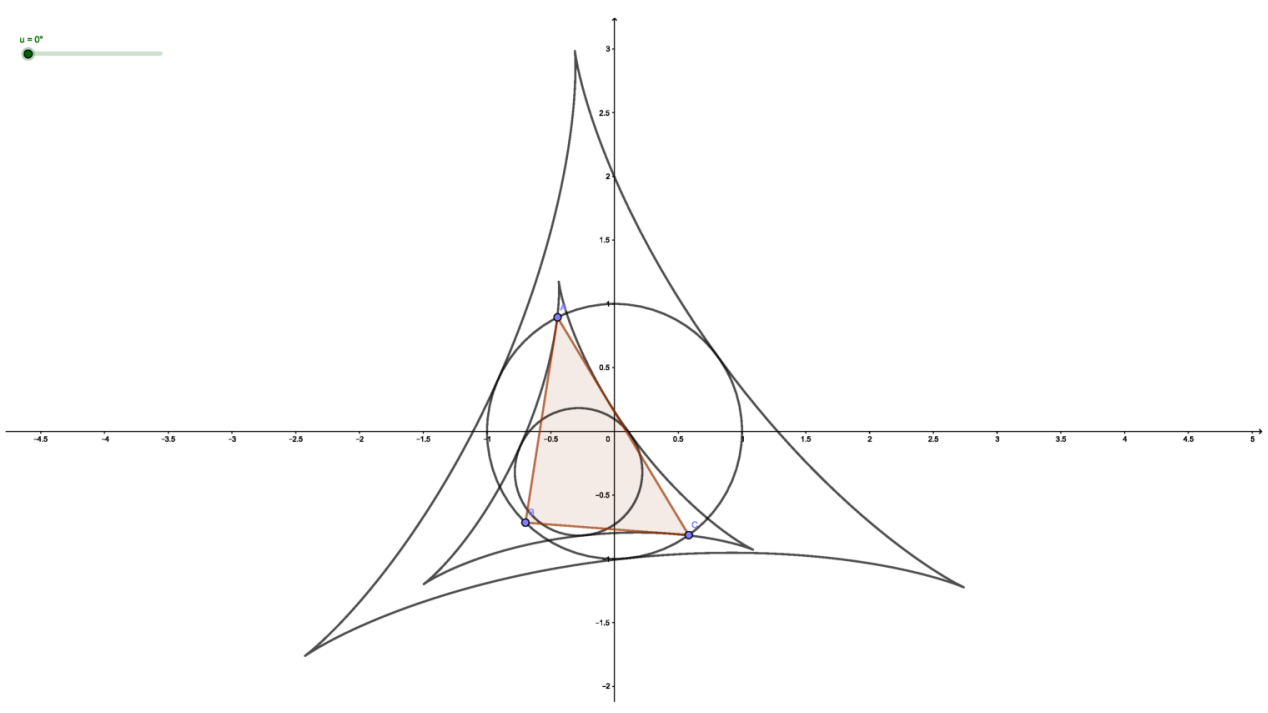

Figure 5. The two deltoids

\section{ACKNOWLEDGEMENT}

The figures used in this paper were prepared using the GeoGebra software which is licensed under the GNU General Public License (GPL) and is free for personal use.

\section{REFERENCES}

[1] C. B. Boyer: A History of Mathematics, Princeton University Press, Princeton, NJ, 1968.

[2] J. Fauvel and J. Gray: The History of Mathematics: A Reader, Macmilan Press Ltd., 1987.

[3] F. Franklin: On applications of circular coordinates, Amer. J. Math. 12 (1890), 161-190.

[4] R. Goormaghtigh: A Theorem on a Cyclic Polygon, The American Mathematical Monthly, 47, No. 7 (Aug.-Sep., 1940), 466-468.

[5] F. Morley: Extensions of Clifford's chain-theorem, Amer. J. Math. 51 (1929),465-472.

[6] F. Morley and F. V. Morley: Inversive Geometry, Ginn \& Co., Boston 1933.

[7] A. Spitzbart: A Generalization of Hermite's Interpolation Formula, The American Mathematical Monthly, 67, No. 1 (Jan., 1960), 42-46.

[8] J. Steiner: Über eine besondere Curve dritter Klasse (und vierten Grades), Journal fr die reine und angewandte Mathematik, 53, (1857), p. 231.

Finbarr Holland is Professor Emeritus at his alma mater University College Cork, where he obtained his MSc in 1962. Following a further two years of study at University College, Cardiff, under the direction of Lionel Cooper, he was awarded the $\mathrm{PhD}$ in Harmonic Analysis by the National University of Wales in 1964. He enjoys creating and solving mathematical problems, and contributes to the problem pages of several journals..

Roger Smyth studied at Cambridge University from 1965 to 1969 and then became a research student of Trevor West at Trinity College Dublin. He received his Ph.D. in 1972. Thereafter he moved into Information Technology and worked in Queens University Belfast and the Northern Ireland Department of Health. His primary mathematical interest is Fredholm theory in Banach algebras, but he maintains a recreational interest in simple Euclidean geometry.

(Finbarr Holland) School of Mathematical Sciences, University College Cork

(Roger Smyth) 15 Harberton Avenue, Belfast BT9 6PH, Northern Ireland.

E-mail address: f.holland@ucc.ie, malcolm.smyth@ntlworld.com 